\title{
Numerical Light Dosimetry in Murine Tissue: Analysis of tumor curvature and angle of incidence effects upon fluence in the tissue
}

\author{
Chao Sheng $^{1 *}$, Brian W. Pogue ${ }^{1 \text { \# }}$, Hamid Dehghani ${ }^{1}$, Julia A. O’Hara ${ }^{2}$, P. Jack Hoopes ${ }^{1,3}$ \\ ${ }^{1}$ Thayer School of Engineering, Dartmouth College, Hanover NH 03755 \\ ${ }^{2}$ Department of Diagnostic Radiology, Dartmouth Medical School, Hanover NH 03755. \\ ${ }^{3}$ Department of Surgery, Dartmouth-Hitchcock Medical Center, Lebanon NH 03756
}

\begin{abstract}
In order to better understand light dosimetry issues for photodynamic therapy (PDT), we have used various tumor and normal tissue geometries to develop a diffusion model of light transport in tissues. We hypothesize that tumor tissues with curved surfaces will have significantly different internal fluence distributions, as compared to tissues with flat surfaces. Using a mouse subcutaneous tumor and rear limb muscle model we compared the internal fluence values within the tissue. In addition, numerical simulations for these corresponding tissue geometries and laser light incidence angles were made. Assuming that the relative photon fluence in the tissue can be accurately modeled by the diffusion equation, we used a finite element approach to approximate the distribution inside the tissue. Meshes with different geometries (flat and curved with different curvatures) were used in this study to mimic the tumor and leg geometries of the murine tumors treated in the lab. Results suggest that tissues surface geometries and incidence angle of light can significantly alter the photon fluence inside the tissue. The photon fluence difference for an $8 \mathrm{~mm}$ diameter, curved surface mouse tumor vs. flat muscle tissue can be as high as $20 \%$. In general, the greater the tissues curvature, the greater the potential loss in light fluence is. In summary, our data demonstrates the importance of tissue surface geometry and the incidence angle of light in determining optimal PDT light dosimetry, and indicates that comparisons between tissue geometries must be carried out with attention to differences in the internal optical distribution.
\end{abstract}

\section{INTRODUCTION}

The use of optical radiation in medical physics is important in several fields, such as tissue ablation and coagulation, photodynamic therapy (PDT), optical imaging and near infrared spectroscopy (NIRS). ${ }^{[1]}$ In this study, we will restrict our discussion to PDT, which is being investigated for a possible treatment for tumors, and is currently used clinically for esophageal and lung tumors, as well as non-cancer applications such as age-related macular degeneration. PDT is a phototherapy, a term which includes all treatments that use light to induce reactions in the body which are of benefit to patient. For our purposes, PDT treatment is done with a drug called a photosensitizer, which is being studied in preclinical animal models of tumors. The photosensitizer is injected into mice bearing tumors, and studies are underway to optimize the delivery of the treatment to the tumor tissue. The photosensitizer alone is thought to be harmless at typical therapeutic doses, indicating it has no effect on either healthy or abnormal tissue. However, when laser is directed onto tissue containing the photosensitizer, the photosensitizer becomes activated and the tissue is rapidly destroyed, but only precisely where the light has been directed. Thus, by careful application of the light beam, the technique can be targeted selectively to the abnormal tissue.

In PDT, several properties of the tissue, including oxygen concentration, photosensitizer concentration and light dose determine efficacy. In this study we concentrated on determining factors that influence the exact number of photons delivered into tumor and normal tissues. We hypothesize that tissue curvature has a significant effect on PDT treatment efficacy, such that it is difficult to directly compare the treatment efficacy in curved versus flat tissue geometries, because the amount of light penetrating the surface of the tissue is changed due to reflections and also due to remission out of the tissue.

Since the lasing dose is an important parameter to evaluate the potential for PDT treatment, it's necessary for us to study light fluence at depth in a number of tissue geometries, including, tissues without tumor (flat muscle tissue geometry), tissues with tumor (curved tumor geometry tissue) using the same laser output for all treatments. Here, we develop a numerical diffusion model to simulate several samples with different geometries. Assuming that the photon

\footnotetext{
*Chao.Sheng@dartmouth.edu, Tel 1 (603) 646-2859, Fax 1 (603) 646-3856

\#Brian.Pogue@dartmouth.edu, Tel 1 (603) 646-3861 Fax 1 (603) 646-3856
} 
transport in the tissue is matched qualitativey to the diffusion equation, we used a finite element solution to approximate the photon fluence inside the tissue. Several meshes with different geometries (flatter, curved with different curvatures) were built. We systematically studied the effects of tissue geometry and angle of incidence on the tumor surface. Experimental studies in tumor tissue were carried out to examine the match to diffusion theory prediction. The implications for these studies for assessment of tumor damage assays compared to normal tissue damage assays are discussed. The results of our simulation and experimental (in vivo) findings were compared and discussed.

\subsection{Diffusion equation}

\section{METHODS}

${ }^{[2,3]}$ Photon transport in tissue maybe modeled in one of two basic ways: an essentially discrete model of individual photon interactions, such as Monte Carlo, or a continuous model based on a differential equation approximation, such as the diffusion equation. ${ }^{[4]}$ Under the assumption that scattering dominates absorption in a region of interest, the Boltzman transport equation can be simplified to the diffusion approximation which in the steady state domain is given by:

$$
-\nabla \cdot \kappa(r) \nabla \Phi(r)+\mu_{a} \Phi(r)=q_{0}(r)
$$

where $q_{0}(r)$ is an isotropic source, $\Phi(r)$ is the photon fluence at position $r$, and

$$
\kappa=\frac{1}{3\left(\mu_{a}+\mu_{s}^{\prime}\right)} \text { is the diffusion }
$$

coefficient. Here the transport scattering coefficient is given by $\mu_{s}{ }^{\prime}=\mu_{s}(1-g)$, where $g$ is the mean cosine of the single scatter function (the anisotropy factor) and $\mu_{s}$ is the scattering coefficient, defining the probability per unit pathlength of a scattering event taking place.

\subsection{Finite element method}

${ }^{[5-8]}$ The finite element method is so widely used for the solution of partial differential equations in complex geometries that here we do not discuss it. The assumption in the FEM approach is that $\Phi$ is approximated by the piecewise polynomial function

$$
\Phi^{h}(r)=\sum_{i}^{d} \Phi_{i} u_{i}(r) \in \Omega^{h}
$$

This function, $u_{i}$ is also known as the interpolation function, shape function or basis function. The aim is to choose a shape function, such that we minimize the error between the actual field $\Phi$ and the calculated field $\Phi^{h}$.

\subsection{Fresnel equations}

Considering the optical reflection that could occur as light getting through the skin, we use the Fresnel equations to derive a better approximation of the light source beneath the skin instead of the laser output. We should determine the reflection and refraction of a mononchromatic plane wave of arbitrary polarization incident at a planar boundary (for curved geometry, we also consider the local geometry as a planar boundary ) between two dielectric media. The media are assumed to be linear, homogeneous, isotropic, nondispersive, and nonmagnetic; the refractive indices are $n_{1}$ and $n_{2}$. The incident, refracted, and reflected waves are labeled with the subscripts $1,2,3$, respectively.

As we know, the wavefronts of these waves are matched at the boundary if the angles of reflection and incidence are equal, $\theta_{3}=\theta_{1}$, and the angles of the refraction and incidence satisfy Snell's law,

$$
n_{1} \theta_{1}=n_{2} \theta_{2}
$$

And we have the following expressions for the reflection of TE and TM polarizations seperately, known as the Fresnel equations:

$$
\begin{aligned}
r_{x}=\frac{n_{1} \cos \theta_{1}-n_{2} \cos \theta_{2}}{n_{1} \cos \theta_{1}+n_{2} \cos \theta_{2}} & \text { TE Polarization } \\
r_{y}=\frac{n_{2} \cos \theta_{1}-n_{1} \cos \theta_{2}}{n_{2} \cos \theta_{1}+n_{1} \cos \theta_{2}} & \text { TM Polarization }
\end{aligned}
$$

From the Fresnel equations, we can easily derive the reflction coefficient $R$ : 


$$
R=\sqrt{r_{x}{ }^{2}+r_{y}{ }^{2}}
$$

and the source beneath the skin will be modified to:

$$
S=S_{0}(1-R)
$$

here, $S_{0}$ is the original output from the laser. And $\mathrm{S}$ is the light fluence after the light has passed through the skin.
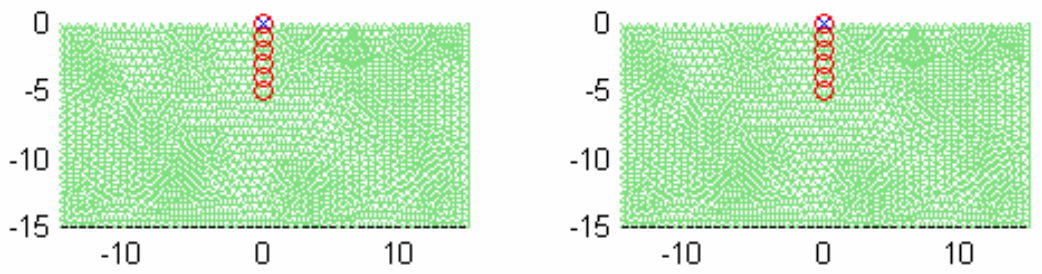

(a) Normal muscle tissue mesh
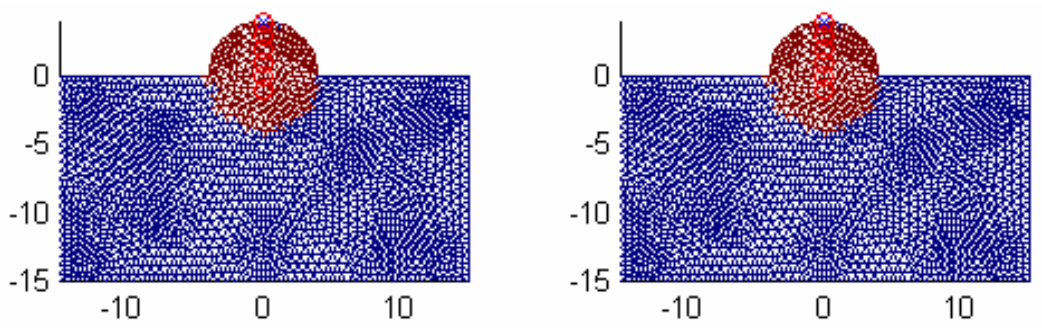

(b) Tumor tissue mesh, geometry type I
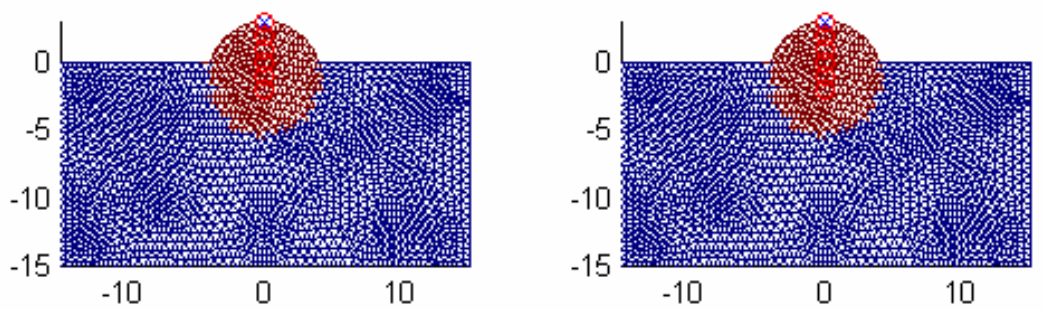

(c) Tumor tissue mesh, geometry type II
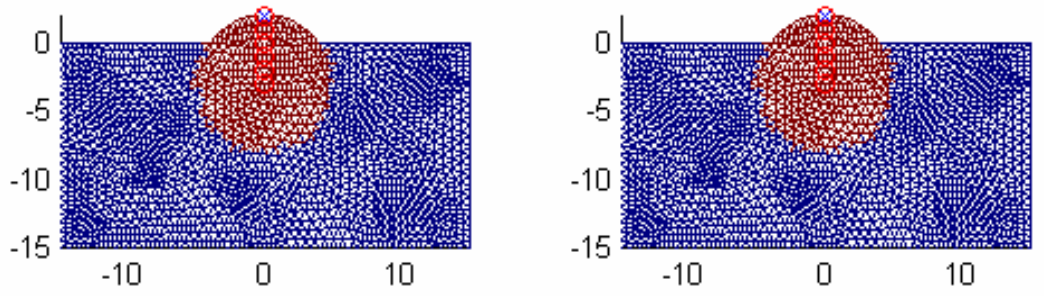

(d) Tumor tissue mesh, geometry type III

Figure 1: The simulation meshes with different geometry types. (a) The normal flat muscle tissue mesh with 1435 nodes and 2736 elements; (b) the tumor geometry type I mesh with 1501 nodes and 2848 elements (circle center $(0,0)$, radius $4 \mathrm{~mm})$; (c) the tumor geometry type II mesh with 1477 nodes and 2816 elements (circle center $(0,-1.167)$, radius $4.167 \mathrm{~mm})$; (d) the tumor type III mesh with 1445 nodes and 2752 elements (circle center $(0,-3)$, radius $5 \mathrm{~mm}$ ). 


\subsection{Simulations}

Four types of slab meshes with the width of $30 \mathrm{~mm}$ and height of $15 \mathrm{~mm}$ were created to do this numerical experiment. As shown in Figure 1 (a-d), one mesh is for flat muscle tissue without tumor, the others are normal tissue with curved geometry tumors, but the tumors have different radius of $4 \mathrm{~mm}, 4.167 \mathrm{~mm}$ and $5 \mathrm{~mm}$, and the widths of the tumor above the slab are all $8 \mathrm{~mm}$. The laser source is centered and has a diameter of $8 \mathrm{~mm}$ to cover the part of the tumor above the slab, and the laser output is normalized to 1 . The optic properties for the normal tissue, tumor tissue and skin are listed in Table -1 . We simulate two types of experiments to assess the different photons transport under the condition of different geometries and different laser incidence angles $\left(0^{\circ}-30^{\circ}\right)$.

\begin{tabular}{|l|c|}
\hline Wavelength & $670 \mathrm{~nm}$ \\
\hline $\mathrm{n} \quad$ (air) & 1 \\
\hline $\mathrm{n} \quad$ (muscle tissue) & 1.33 \\
\hline $\mathrm{n}$ (tumor tissue) & 1.33 \\
\hline $\mathrm{n}$ (skin) & 1.45 \\
\hline$\mu_{\mathrm{a}}$ (muscle tissue) & 0.01 \\
\hline$\mu_{\mathrm{s}}{ }^{\prime}$ (muscle tissue) & 1.0 \\
\hline$\mu_{\mathrm{a}}$ (tumor tissue) & 0.017 \\
\hline$\mu_{\mathrm{s}}{ }^{\prime}$ (tumor tissue) & 1.04 \\
\hline
\end{tabular}

Table 1: Optical properties used in the simulations. Here, $\mathrm{n}$ is the refractive index of different media. $\mu_{a}$ is the absorption coefficient, and $\mu^{\prime}$ is the ransport scattering coefficient.

\subsection{Murine Experiments (in vivo)}

All experiments were carried out under animal protocols approved by the Institutional Animal Care and Use Committee (IACUC), and animals were housed and cared for in the Animal Resource Center at Dartmouth Medical School, Dartmouth College. C3H mice were ordered from Jackson Labs (ME) and used for subcutaneous implantation of MTG-B tumor cells in the hind right flank of the animal.

Five mice with subcutaneous MTGB murine tumors were used to assess the photon attenuation in the tumor. A cut end fiber with the diameter of $200 \mu \mathrm{m}$ was inserted into the tumor at different depth from $0.5 \mathrm{~mm}$ to $6.75 \mathrm{~mm}$ with the interval of $0.625 \mathrm{~mm}$. The use of a cut end fiber was justified as a way to same a specific solid angle of the isotropic fluence within the tissue. At distances further from the source, this isotropic flux is thought to be a good predictor of the total fluence in the tissue. The light from the fiber was detected by a sensitive Silicon photodiode-based power meter (Newport Instruments). All the flux data were normalized to the data at the skin. Assuming that the light is isotropic at each point inside the tumor, we can calculate the photon fluence according to the light flux recorded and normalize it. The slop of this line at deeper depths in the tissue is related to the effective attenuation coefficient of the tissue.

\section{RESULTS}

Assuming the incident light is equally distributed, the numbers of photons delivered to the curved and flat tissue are significantly different (Figure 2, 4 and 5). At an incidence angle of $0^{\circ}$, the ratios of photon fluence of tumors to the flat tissue are $70.7 \%, 76.3 \%$ and $76.7 \%$, for the three tumor curvature values denoted by types I II III, respectively in Figure 1. With the incidence angle of $30^{\circ}$, the ratios of photon fluence of tumors to the flat tissue are $59.5 \%, 62.5 \%$ and $68.5 \%$, again for the three tumor types I II III, respectively.

When we change the incidence angle between $0^{\circ}$ to $30^{\circ}$, shown in Figures 4 and 5, the total photons delivered into the flat tissue is changed by less than $1 \%$ from 224.2 to 222.5 . For the tumor type I, the fluence is changed by $16 \%$ from 158.5 to 132.6. For the tumor type II, the fluence is changed by $19 \%$ from 171.1 to 139.2 . For the tumor type III, the fluence is changed by $11 \%$ from 172.0 to 152.5 . Thus the changing of incidence angle can make more difference in the photon fluence in tumor than the flat tissue, which can become a major issue for typical animal experiments where the tissue geometry is quite often irregular. 

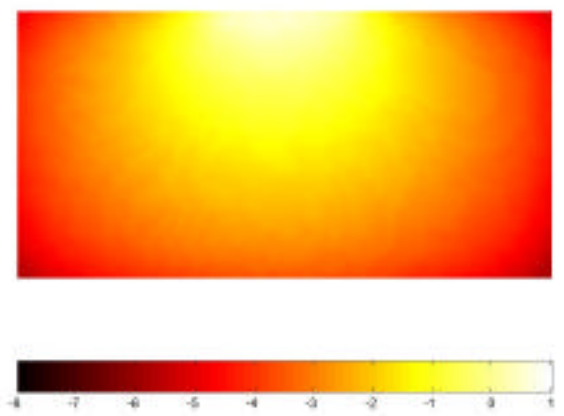

(a)

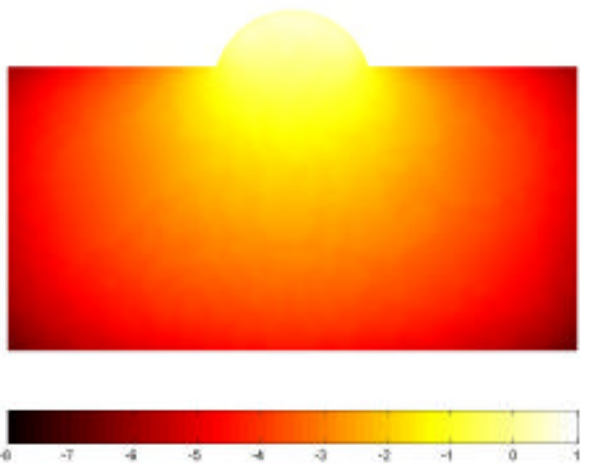

(c)
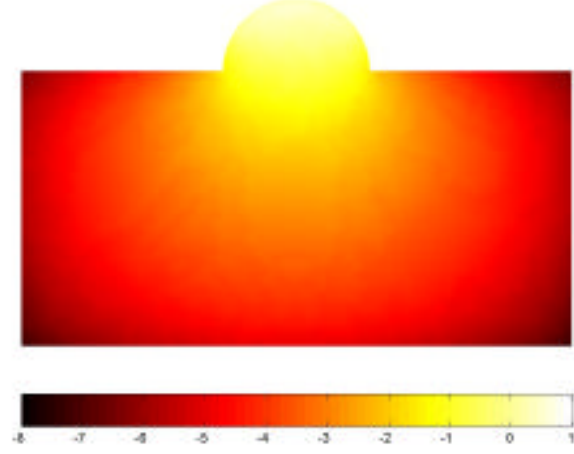

(b)

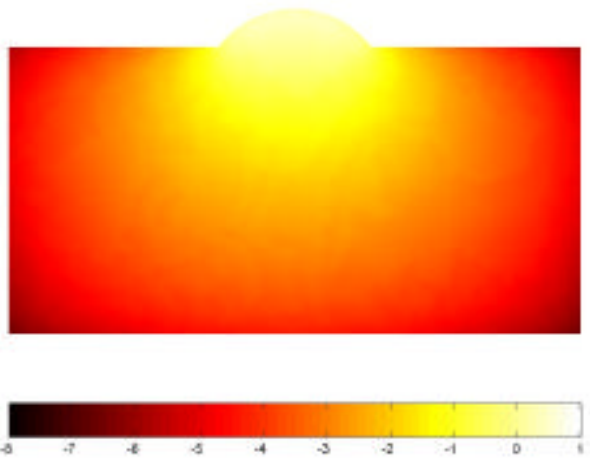

(d)

Figure 2: Effect of geometry on photon fluence in the simulation. The light incidence angle is $0^{\circ}$. All the photon fluence data shown in the figure is in the log format, the colormap varies from -8 to 1 . (a) is the flat muscle tissue, (b) is the tumor tissue of geometry type I, (c) is the tumor tissue of geometry type II, (d) is the tumor tissue of geometry type III. (illustrated in Figure 1). 


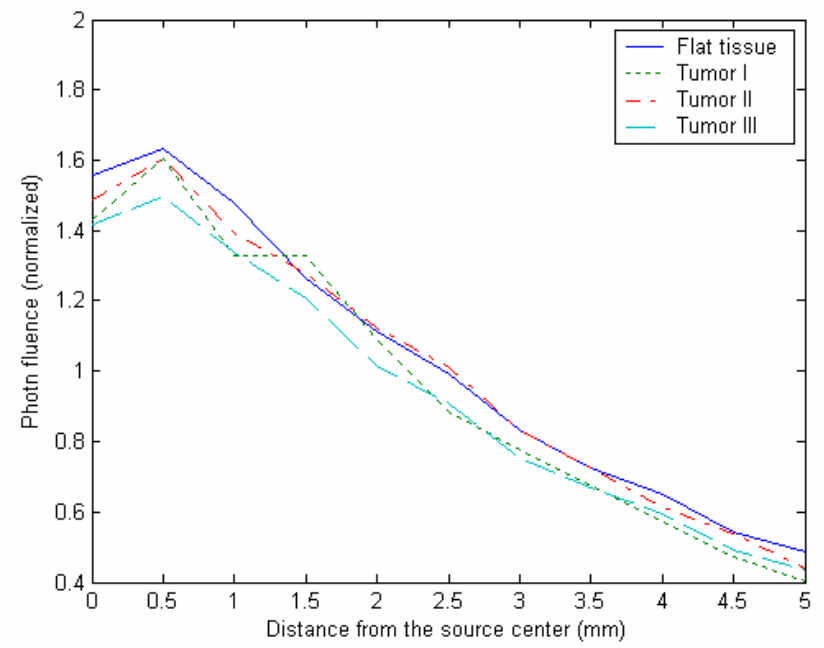

Figure 3: The photon fluence in the simulation attenuates along a line below the source center from $0 \mathrm{~mm}$ to $5 \mathrm{~mm}$. The light incidence angle is $0^{\circ}$. Assume the incidence light has the fluence of 1 . All the sample points are beneath the skin.

Figure 3 shows that in the simulation the photon fluence attenuation along the line below the source, we find that the photon fluence attenuates quickly within $5 \mathrm{~mm}$. Different geometries cause different absolute photon fluence values, but the attenuate rates are almost same between different geometries.
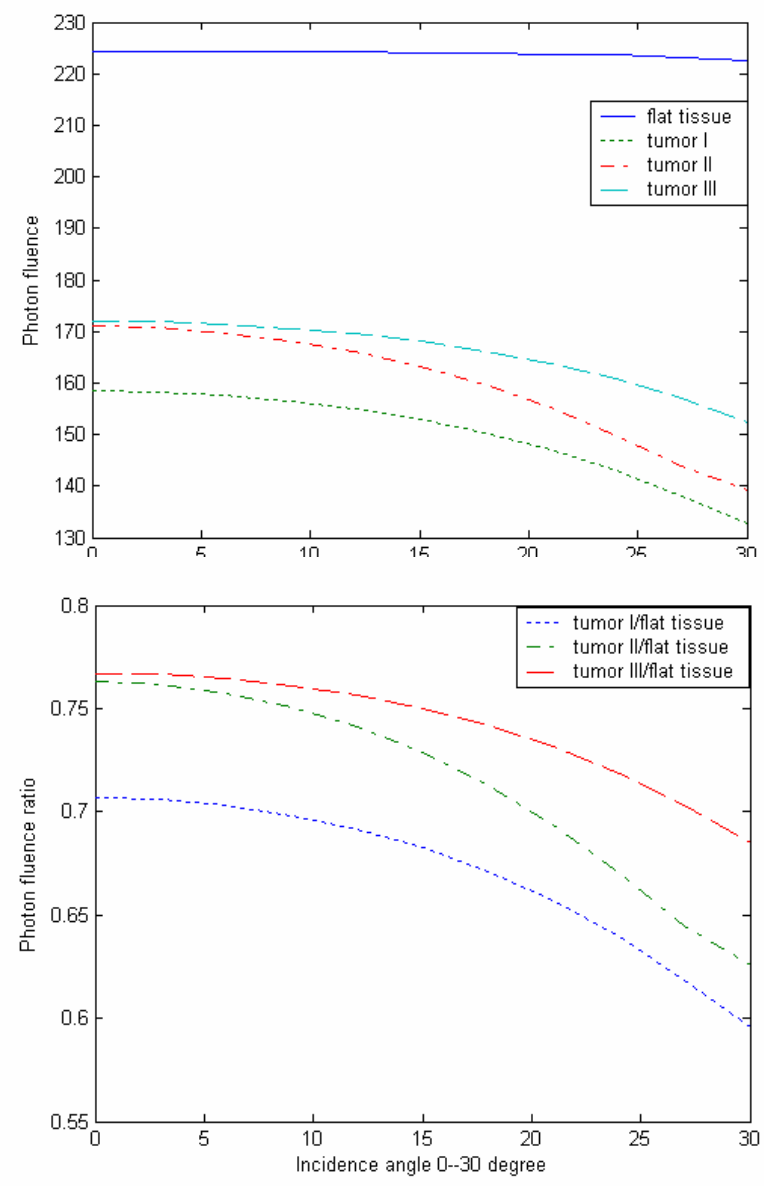

Figure 4: The photon fluence in the whole simulation area changes with the incidence angle. As the incidence angle increase from $0^{\circ}$ to $30^{\circ}$, the photon fluence in the flat muscle tissue changed from 224.2 to 222.5 , the photon fluence in the tumor type I changed from 158.5 to 132.6 , the photon fluence in the tumor type II changed from 171.1 to 139.2 , the photon fluence in the tumor type III changed from 172.0 to 152.5 .

Figure 5: Photon fluence ratios in the simulation change along with the increasing of incidence angle from $0^{\circ}$ to $30^{\circ}$. For the incidence angle of $0^{\circ}$, the ratios of photon fluence of tumors to the flat tissue are $70.7 \%, 76.3 \%$ and $76.7 \%$, for the three tumor curvature values denoted by types I II III. The ratios drop to $59.5 \%, 62.5 \%$ and $68.5 \%$ when the incidence angle increased to $30^{\circ}$. 


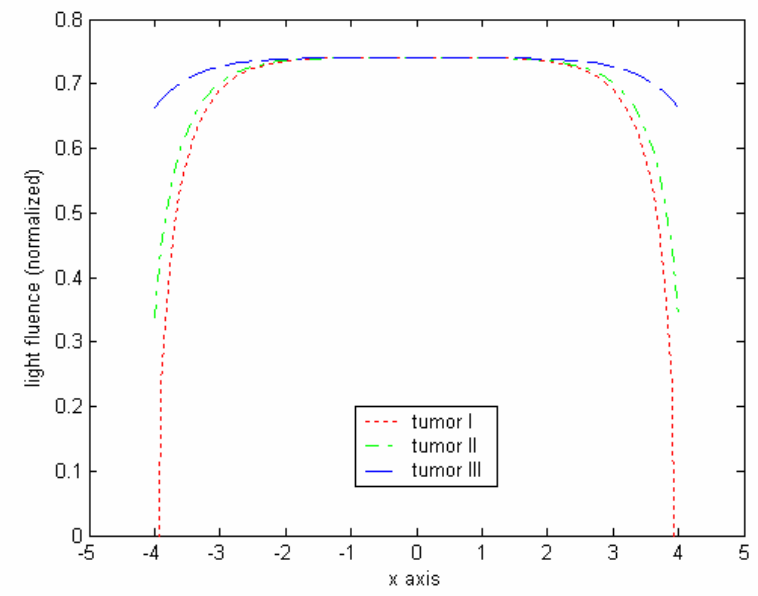

Figure 6: The light fluence in the simulation after including the skin reflection along the $\mathrm{x}$-axis. This figure shows the attenuation of the light as it passes through the skin. Assume the light incidence fluence is 1 and the tumor diameter is $8 \mathrm{~mm}$. The dominant reflections occur at the edge of the tumor. The tumor with larger geometry curvature has more light attenuation because of the skin reflection.

Considering the reflection of the skin in the simulation, we find the reflection is quite different within different tumor geometries. With the same incidence light fluence of 1 , the dominant reflection occurs at the edge of the tumor. The larger geometry curvature the tumor has, the more light fluence attenuation would caused by the reflection.

Shown in Figure $7(\mathrm{a}-\mathrm{b})$, the MTGB tumor radius of $5 \mathrm{~mm}$, we normalized all the data to the data recorded $0.5 \mathrm{~mm}$ beneath the skin. The photon fluence was attenuated to near $21 \%$ at a depth of $6.75 \mathrm{~mm}$ under the skin after the light getting through the skin. From the diffusion approximation, the photon fluence was attenuated to $17 \%$ at the depth of $6.75 \mathrm{~mm}$ under the skin. The simulations gave relative fluence values that were very close to the experimental relative data.

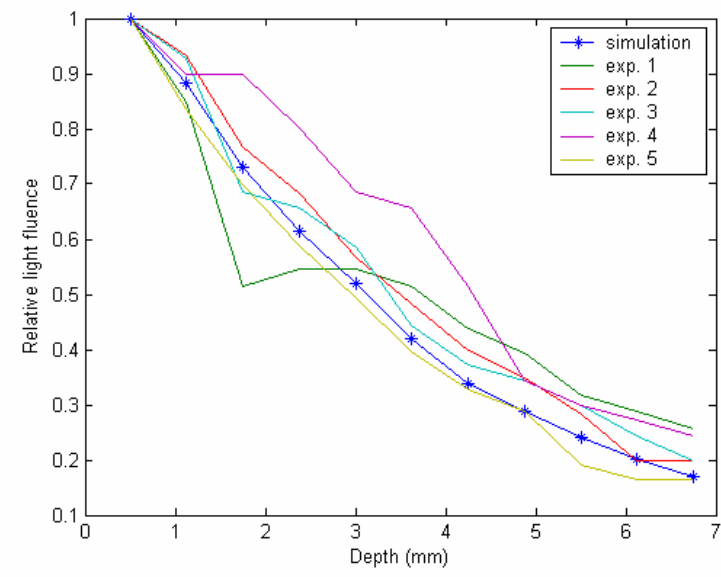

(a)

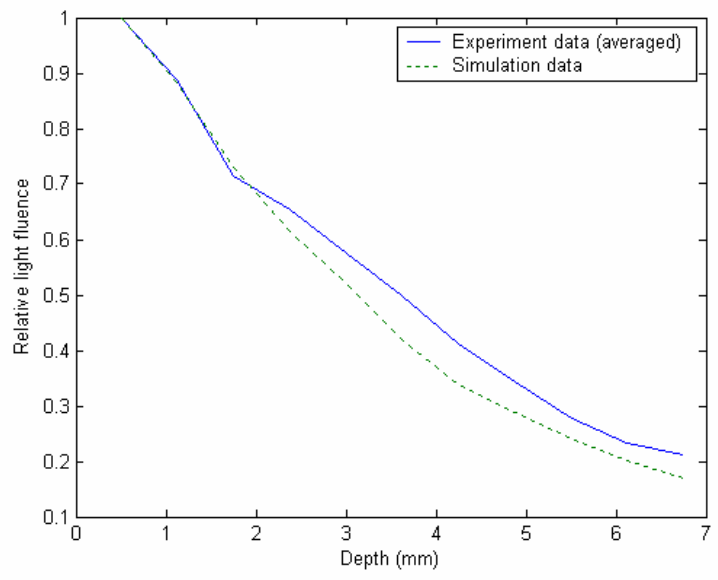

(b)

Figure 7: The light fluence attenuation by the simulation and murine experiment. (a) 5 mice were used for the experiment, the light fluence was measured at the depth from $0.5 \mathrm{~mm}$ to $6.75 \mathrm{~mm}$. All the data are normalized to the first measure point for each mouse. (b) The averaged data showed the photon fluence was attenuated to near $21 \%$ at a depth of $6.75 \mathrm{~mm}$ under the skin after the light getting through the skin. From the simulation, the photon fluence was attenuated to $17 \%$ at the depth of $6.75 \mathrm{~mm}$ under the skin. 


\section{DISCUSSION}

In this study, we find that curved geometry tumor tissue and the flat muscle tissue have quite different photon fluence with the same laser output, which means when we use the same laser output and lasing time for PDT study, the exact light delivered into the area of interest in flat muscle tissue and the tumor are significantly different. If unaccounted for, this difference could alter the evaluation of how PDT treatment affects tumor versus normal tissues. If we want to make the evaluation more precise, we should adjust the output of the laser or the lasing time for different tissue geometry (flatter or curved).

When we measured the photon fluence below the source point (shown in Figure 7), the attenuation curve of the tumor is almost same as the in vivo experiment data (i.e. having the same shape and same attenuation rate). This match between the simulation and the experiment was fortunate. In general, it is well known that diffusion theory prediction cannot be assumed to be accurate for measurements less than 3 scattering lengths from the source position, so we might expect the maximum mismatch to be near the surface of the tumor. However in our simulations, we found good matching between simulation and experiment. This was encouraging and may form the basis for further use of diffusion theory in these tumors. However, it is important to use caution when interpreting these predictions since the limitations of diffusion theory prediction are well known in these small tissue geometries.

We also studied the reduction in source intensity below the skin, due to loss of light from reflection off of the top surface of the tissue. From Figure 6 we find that the dominant reflections occur at the edge of the tumor as might be expected since this is where the greatest curvature exists. The reflection coefficient changes a lot due to the different geometries (curvature and position of the center of the circle), making reflections off of a tumor much more problematic than reflections off of flat normal tissues. The incidence angle does alter the photon fluence inside the tissue. It is important to make the laser perpendicular to the tumor, in order to minimize transmission errors in animal studies with small tumors. But in the flat tissue, the incidence angles do not make such big influence. And we can also find it from the results that if the incidence angle is below the $15^{\circ}$, the difference is below $5 \%(3.8 \%, 4.7 \%$, and $1.8 \%$ for tumor type I II III, as shown in Figure 1 respectively). So, if the incidence angle is quite smaller than $15^{\circ}$ in the PDT treatment, it is not necessary to adjust the incidence angle.

\section{CONCLUSIONS}

We have introduced a finite element method for deriving photon fluence inside an object, assuming that the photon transport model is the diffusion approximation to the Boltzman transport equation. We have given results from the model for a particular case: the calculation of the photon fluence inside different tissue geometries. With this study of numerical light dosimetry, We have demonstrated the difference of the photon fluence between different tissue geometries with same laser output.

Undoubtedly more study is required to determine the applicablity of this model, such as Monte Carlo method and 3-D model. But once these studies have been done, we are confident it should be widely adopted to improve the procedures used for studies in the treatment of pre-clinical animal tumor models.

\section{ACKNOWLEDGEMENTS}

This work has been sponsored by the NIH through grants R44 CA76913, RO1 CA78734 and PO1 CA84203.

\section{REFERENCES}

1. B. C. Wilson and S. L. Jacques, “ Optical reflectance and transmittance of tissue: Principles and applications," IEEE J. Quantum Electron. 26(12), 2186-2199 (1990).

2. M. S. Patterson, S. J. Madsen, J. D. Moulton and B. C. Wilson, “ Diffusion equation representation of photon migration in tissue”, IEEE MTT-S Digest, 905-908, 1991. 
3. T. J. Farrell, M. S. Patterson and B. C. Wilson, "A diffusion theory model of spatially resolved, steady-state diffuse reflectance for the noninvasive determination of tissue optical properties in vivo" , Medical Physics, Vol. 19, No.4, Jul/Aug, 879-888,1992

4. M. S. Patterson, B. C. Wilson and D. R. Wyman, "The propagation of optical radiation in Tissue 1: Models of radiation transport and their application," Laser Med. Sci. 6, 155-168 (1991).

5. S. R. Arridge, M. Schweiger, M. Hiraoka and D. T. Delpy, "A finite element approach for modeling photon transport in tissue" , Medical Physics, Vol. 20, N0. 2, Pt. 1, Mar/Apr, 299-309, 1993.

6. M. Schweiger, S. R. Arridge, M. Hiraoka and D. T. Delpy, " The finite element method for the propagation of light in scattering media: Boundary and source conditions”, Med. Phys. 22(11), 1779-1792 (1995).

7. B. W. Pogue, S. Geimer, T. Mcbride, S. Jiang, U. L. Osterberg, and K. D. Paulsen, "3-d simulation of near-infrared diffusion in tissue: boundary conditions and geometry analysis for a finite element reconstruction algorithm," applied optics, vol.40, pp. 588-600, 2001

8. H. Dehghani, B. W. Pogue, S. P. Poplack, K. D. Paulsen, "Multiwavelength Three-Dimensional Near-Infrared Tomography of the Breast: Initial Simulation, Phantom, and Clinical Results", Applied Optics Volume 42, Issue 1, pp. 135-145 (2003)

9. T. Johansson, M. S. Thomp son, M. Stenberg, C. af Klinteberg, S. Andersson-Engels, S. Svanberg and K. Svanberg, "Feasibility study of a system for combined light dosimetry and interstitial photodynamic treatment of massive tumors", Applied Optics, Vol. 41, N0. 7, 1 Mach 2002.

10. B. C. Wilson, P. J. Muller and J. C. Yanch, " Instrumentation and light dosimetry for intra-operative photodynamic therapy (PDT) of malignant brain tumors", The Institute of physics, 1998. 